\title{
Moisture Management Performance of Multifunctional yarns based on Wool Fibers
}

\section{Raul Manuel Esteves Sousa Fangueiro ${ }^{1, ~ a, ~ H e ́ l d e r ~ F i l i p e ~ d a ~ C u n h a ~ S o u t i n h o ~}{ }^{2, b}$ and Carla Freitas ${ }^{3, c}$}

${ }^{1}$ School of Engineering, University of Minho, 4800-058 Guimarães, Portugal

${ }^{2}$ School of Engineering, University of Minho, 4800-058 Guimarães, Portugal

${ }^{3}$ Fiação da Graça SA, Lugar da Veiga, 4700-670 Padim da Graça, Portugal

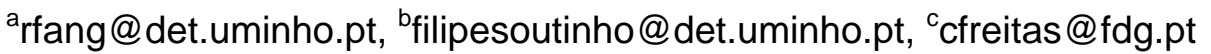

Keywords: Drying capability, Functional yarns, Wool, Wicking performance.

\begin{abstract}
Blends of wool and moisture management fibres such as Coolmax and Finecool have been prepared to produce innovative yarns with specific functionalities. These yarns have been used to produce knitted fabrics and their performance was evaluated, including vertical and horizontal wicking. The influence of wool fibre proportion on the performance of each blend is analyzed. It is observed that the Coolmax based fabrics show the best capillarity performance, and the wool fibre based fabrics show lower water absorption performance.
\end{abstract}

\section{Introduction}

Wool remains a premium, apparel fibre with an impressive set of technical attributes. For carpets and rugs, wool remains the benchmark of quality and performance with which other fibres are compared [1].

The unique physical and chemical structures of wool create a range of natural characteristics that have been proved ideal for apparel, upholstery fabrics and carpets [2]. These characteristics are: warmth and coolness, breathability, moisture absorption and buffering, resilience, low odour, odour absorption, softness, flame resistance, biodegradability and recyclability, resistance to soiling and staining [2]. These special characteristics of wool can be exploited in a worsted suit or knitted outerwear or enhanced to create novel apparel fabrics. Wool is increasingly being used in technical applications in which its unique properties and the opportunities for specific enhancements can be profitably utilised [1].

The versatility of wool has been demonstrated by a number of innovative apparel products entering the sportswear marketplace. These products, which combine different fibres and structures, include the Icebreaker and SportWool ranges.

Liquid transporting of fabrics is one vital factor affecting the physiological comfort of garments [3 -5]. The moisture transfer behaviour of textiles depends mainly on the capillary capability and moisture absorbency of their fibers. Wicking is the spontaneous flow of the liquid in a porous substance, driven by capillary forces. Washburn [6] proposed the well-known Lucas-Washburn kinetics equation to describe the relationship between wicking length and wicking time. Crow and Randall, Kissa, Weiyuan et al [7 -9] investigated wetting and wicking behaviour of textiles. As capillary forces are caused by wetting, wicking is a result of spontaneous wetting in a capillary system. Wicking takes place only in wet fabrics and the contact angle decides the wicking behaviour. 


\section{Materials and Methods}

Materials. Wool fibres $(19 \mu)$ with Basolan treatment were combined in different proportions (Table 1) with moisture management fibres: Finecool (2.4 dtex) and Coolmax (2.4 dtex). Yarns with $100 \%$ of the same fiber type, blends with $50 \%$ of wool and $50 \%$ of moisture management fibres, and blends with $75 \%$ of wool and $25 \%$ of moisture management fibers have been developed and produced. All the yarns produced present a linear density of 20 tex with 630 turns/m of twist. Table 1 shows the dimensional properties of single jersey knitted fabrics, produced on a circular weft-knitting machine (E28 gauge) with the yarns developed.

\begin{tabular}{ccccc}
\hline \multicolumn{4}{c}{ Table $1-$ Dimensional properties of the knitted fabrics produced } \\
Fabric & $\begin{array}{c}\text { Cover Factor } \\
\text { Aerial mass } \\
{[\mathrm{K}]}\end{array}$ & $\begin{array}{c}\text { Density } \\
{\left[\mathrm{g} / \mathrm{m}^{2}\right.}\end{array}$ & $\begin{array}{c}\text { Thickness } \\
{[\mathrm{mm}]}\end{array}$ \\
$\begin{array}{c}\text { Wool } \\
\text { Polyester }\end{array}$ & 15.68 & 155.23 & $16 \times 20$ & 0.68 \\
Wool/Polyester & 16.86 & 168.73 & $14 \times 22$ & 0.67 \\
$\quad$ (50:50) & 16.28 & 147.67 & $14 \times 20$ & 0.64 \\
$\begin{array}{c}\text { Finecool } \\
\text { Wool/Finecool }\end{array}$ & 16.64 & 158.91 & $14 \times 21$ & 0.71 \\
$\quad$ (50:50) & 15.79 & 164.11 & $15 \times 19$ & 0.66 \\
$\begin{array}{c}\text { Wool/Finecool } \\
\text { (75:25) }\end{array}$ & 17.12 & 161.53 & $16 \times 19$ & 0.68 \\
$\begin{array}{c}\text { Coolmax } \\
\text { Wool/Coolmax }\end{array}$ & 16.40 & 163.49 & $15 \times 19$ & 0.63 \\
$\begin{array}{c}\text { (50:50) } \\
\text { Wool/Coolmax } \\
(75: 25)\end{array}$ & 16.18 & 154.68 & $14 \times 20$ & 0.61 \\
\hline
\end{tabular}

Methods. For vertical wicking five specimens of 200X25mm size cut along wale-wise and coursewise directions were prepared. The specimens were suspended vertically with their bottom ends dipped in a reservoir of distilled water. In order to ensure that the bottom ends of the specimens can be immersed vertically with $30 \mathrm{~mm}$ depth into the water, the bottom end of each specimen was clamped with a clip. The wicking heights, measured after every 30 seconds till 5 min, were recorded as a direct evaluation of the fabric wicking ability.

The horizontal wicking rate is evaluated under a standard environment at conditions $\left(20 \pm 2^{\circ} \mathrm{C}\right.$ and $65 \pm 2 \% \mathrm{RH})$. In the horizontal wicking apparatus, a tiny water drops contact with the horizontally placed specimen $(200 \mathrm{~mm} \times 200 \mathrm{~mm})$ was provided, leading to the water absorption by wicking and wetting through the pores. The water was supplied continuously from a reservoir by siphoning. The reservoir was kept on an electronic balance, which enables to record the water mass absorbed by the fabric. Because the mass absorbed by the sample is related to the sample thickness, water absorption per unit of thickness is used to evaluate the horizontal wicking ability. The wicking was measured after every minute till $10 \mathrm{~min}$.

\section{Results and Discussion}

Horizontal Wicking. Figure 1 shows the results obtained for horizontal wicking tests. Considering that during intense physical activity, when the body starts perspiration, quick moisture transportation is important, the results have been analyzed after the first minute. The performance ranking of fabrics (from the best to the worst performence) is shown below:

Coolmax $>$ Finecool $>$ Polyester $>$ Wool / Coolmax (75:25) $>$ Wool / Coolmax (50:50) $>$ Wool / Finecool (75:25) > Wool / Finecool (50:50) > Wool / Polyester (50:50) > Wool. 
It can be seen that the fabric with $100 \%$ Coolmax fibres shows the best performance. Moreover, the fabrics with $25 \%$ and $50 \%$ of Coolmax show better performance than those with the same amount of Finecool. Fabric with $100 \%$ wool presents the poorest behaviour. For fabrics produced with $100 \%$ of the same material it is possible to easily identify the trends of horizontal wicking behaviour, as the diameter of the fibres in the yarn structure is constant. When different fibres are blended, their diameters vary according to the fibres density, leading to the different arrangements in the yarn and consequently different wicking channels.

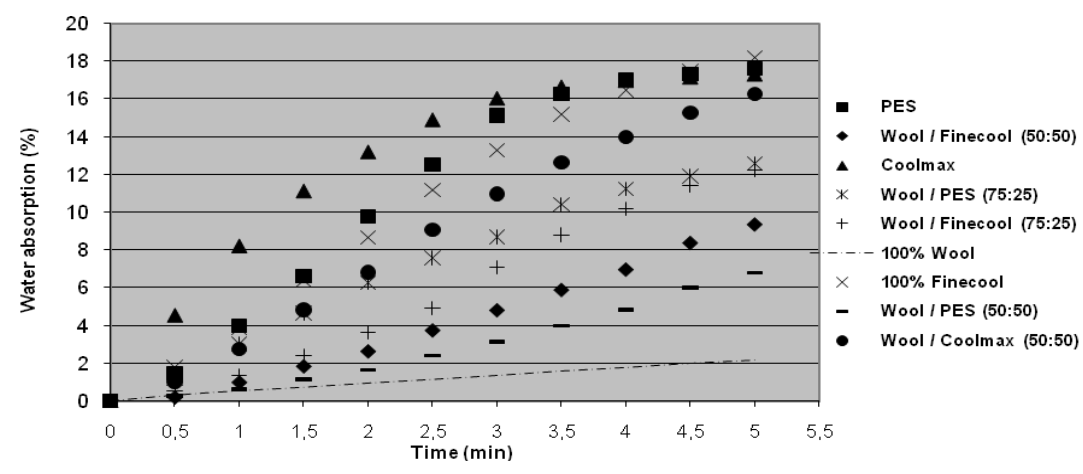

Fig. 1 - Horizontal wicking curves

Vertical Wicking. Figure 2 shows the results obtained for vertical wicking in the course-wise and wale-wise directions. Based on the findings at $1^{\text {st }} \mathrm{min}$, performance ranking (from the best to the worst) is shown below:

Course-wise direction: Coolmax > Polyester > Wool / Finecool (75:25) > Wool / Coolmax $(50: 50)>$ Finecool > Wool / Coolmax (75:25) > Wool / Finecool (50:50) > Wool / Polyester (50:50) > Wool

Wale-wise direction: Coolmax > Polyester > Wool / Finecool (75:25) > Wool / Coolmax $(50: 50)>$ Finecool > Wool / Coolmax (75:25) > Wool / Polyester (50:50) > Wool / Finecool $(50: 50)>$ Wool

It is found that the $100 \%$ Coolmax fabric shows the best performance in both directions, and for all the other fabrics the results are very similar in both course-wise and wale-wise directions. The fabric with $75 \%$ wool and $25 \%$ Finecool gives very good results, better than that of fabric with $100 \%$ Finecool in both directions. 100\% wool fabric shows the poorest results.
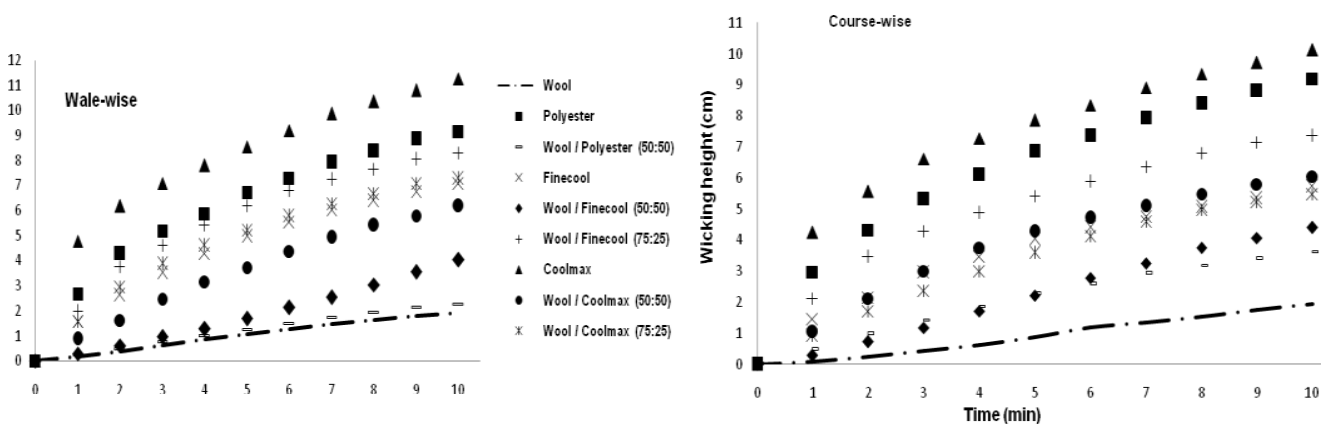

Fig. 2 - Vertical wicking curves

Influence of the Amount of Fibre. Figures 3 and 4 shows the performance of different blends, depending on the different percentages of wool and functional fibres. It is observed that, for horizontal wicking, the increase in the amount of wool in the fabrics leads to a decrease in the absorption. However, the fabrics with $75 \%$ wool and $25 \%$ functional fibre show better performance than that of fabrics with $50 \%$ wool and $50 \%$ functional fibre. In vertical wicking similar conclusions may be observed. However, in this case, fabrics with $75 \%$ wool show poor performance than those of fabrics with $50 \%$ wool. For horizontal wicking, all proportions of functional fiber (100, 75 and 25\%), Coolmax gives better performance. In vertical wicking, the 
trend is similar but fabric with wool/Finecool (75:25) shows the best performance. However the performance is very similar in course-wise and wale-wise directions for all other proportions of different fibres

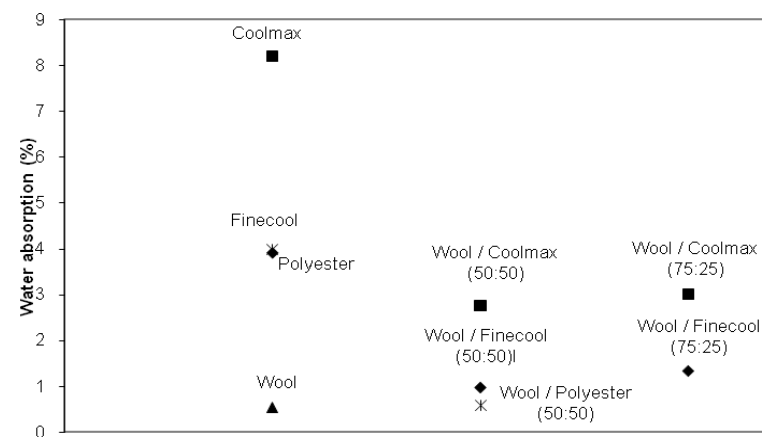

Fig. 3 - Influence of type of fibre for the $1^{\text {st }}$ min. in horizontal wicking
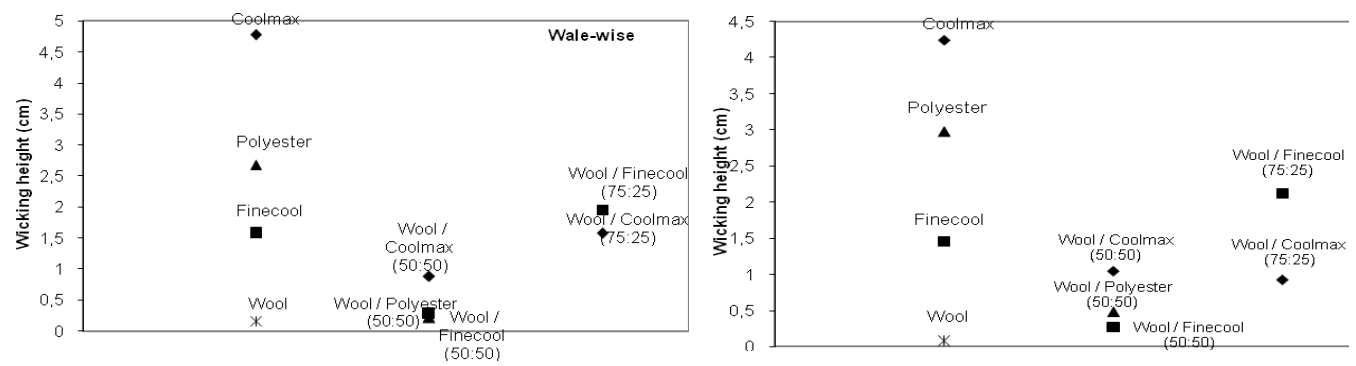

Fig 4 - Influence of the type of fibre for the $1^{\text {st }} \min$ in the vertical wicking

\section{Conclusions}

Wool fiber based fabrics present low water absorption performance. The increase in the percentage of wool fiber in the fabrics is directly related to the decrease of their water absorption performance. The trends found out in this work help in designing the most suitable combination wool/functional fibres for end-uses where moisture management is an important issue. The quantity of functional fibres can also be optimized to produce the most economical products to address specific market demands.

\section{References}

[1] Johnson NAG, Wood E J, Ingham P E, MeNeil S J, \& MeFarlane I D, Wool as a technical fibre, Text Inst, Part 3 (2003), 94.

[2] Sailen K Chaudhuri, Taking wool into the future - Through innovations, paper presented at 21 National Textile Technicians, congress, Brazil, 2004.

[3] Laughlin R D, Davies J E, Some aspects of capillary absorption in fibrous textile wicking, Text Res Journal, 31, Pg. 904-910, (1961).

[4] Norman R S, Hollies Kassinger, Water transport mechanisms in textile material, Part II: Capillary-type penetration in yarns and fabrics, Text Res, 8, (1957), 132.

[5] Yan Zhang \& Huaping Wang, Modelling of capillary flow in shaped polymer fiber bundles, J. Mater Sci., 42, (2007), 1327.

[6] Washburn E W, The dynamics of capillary flow - Physics Review, 1921, 273-283.

[7] Rita M Crow \& Randall J, The Interaction of water with Fabric, Text Res, 68, (1998), 280-288.

[8] Erik Kissa, Wetting and Wicking, Text Res, 9, (1996), 660-668.

[9] Weiyuan Z, Jun L, Wenfei C \& Shilong, Wetness comfort of fine polypropylene fibre fabrics, Text Inst, (1997), 252. 
Multi-Functional Materials and Structures III

doi:10.4028/www.scientific.net/AMR.123-125

Moisture Management Performance of Multifunctional Yarns Based on Wool Fibers doi:10.4028/www.scientific.net/AMR.123-125.1247 